\title{
CENTERS OF HYPERGROUPS ${ }^{1}$
}

BY

\author{
KENNETH A. ROSS
}

\begin{abstract}
This paper initiates a study of $Z$-hypergroups, that is, commutative topological hypergroups $K$ such that $K / Z$ is compact where $Z$ denotes the maximum subgroup (equivalently, the center) of $K$. The character hypergroup $K$ is studied and its connection with the locally compact abelian group $Z$ is given. Each $Z$-group is shown to correspond in a natural way to a $Z$-hypergroup. It is observed that the dual of a $Z$-group is itself a hypergroup. The basic orthogonality relations on $Z$-groups due to $S$. Grosser and M. Moskowitz are shown to hold for most Z-hypergroups. Some results on measure algebras of compact hypergroups due to C. F. Dunkl are extended to a class of noncompact hypergroups.
\end{abstract}

1. Introduction. The theory of topological hypergroups was initiated by Dunkl [3], Jewett [8] and Spector [14] and has recently received a good deal of attention from harmonic analysts. This is because the setting of hypergroups is sufficiently general to cover a variety of important examples including double-coset spaces, and yet is concrete enough to allow a reasonable theory to develop. A fairly complete history is given in [12].

Our goal here is to initiate a study of $Z$-hypergroups, which are analogous to $Z$-groups. Each $Z$-group corresponds in a natural way to a $Z$-hypergroup [§5]. The basic orthogonality relations on $Z$-groups due to Grosser and Moskowitz [6] hold also for most Z-hypergroups [\$4]. Along the way [\$3] we will generalize some results of Dunkl [3] concerning the center of a hypergroup. We also indicate in $\$ 6$ how his results on measure algebras extend to a class of noncompact hypergroups. In $\$ 2$ we set down some useful basic results that one would expect to hold.

Throughout this paper $K$ will denote a commutative hypergroup. Our basic reference will be Jewett [8], who called hypergroups "convos". In particular, we adopt Jewett's axioms, which differ somewhat from those of Dunkl and Spector. Unless otherwise specified, our notation will follow that of Jewett. We will use $x \mapsto \check{x}$ instead of $x \mapsto x^{-}$for the involution of $K$.

Received by the editors May 12, 1977.

AMS (MOS) subject classifications (1970). Primary 22A99, 43A10.

Key words and phrases. Hypergroup, center of hypergroup, character hypergroup, Z-group, automorphism group, orthogonality relations, measure algebras.

'Financial assistance by the National Science Foundation Grant MCS 76-07272 is gratefully acknowledged. The author has benefitted from valuable discussions with A. K. Chilana, D. K. Harrison and T. W. Palmer and from some helpful comments by the referee.

- American Mathematical Society 1978 
We now discuss some hypergroups that are induced from groups. Let $G$ be a locally compact [Hausdorff] group and let $B$ denote a subgroup of the automorphism group $\operatorname{Aut}(G)$ that contains the group $I(G)$ of inner automorphisms. We call $G$ an $[F I A]_{B}^{-}$-group provided the closure $B^{-}$of $B$ in $\operatorname{Aut}(G)$ is compact. For each $x$ in $G$, let $[x]$ denote the $B^{-}$-orbit of $x$ in $G$, i.e. $[x]=\left\{\beta(x): \beta \in B^{-}\right\}$. We write $G_{B}$ for the space of all $B^{-}$-orbits; thus $G_{B}$ is exactly $G^{B^{-}}$as defined by Jewett $[8,8.1]$. The space $G_{B}$ is a commutative hypergroup with the operation

$$
p_{[x]} * p_{[y]}=\int_{B^{-}} p_{[\beta(x) y]} d \beta ;
$$

see $[8,8.3 \mathrm{~A}]$. It can be shown that the natural map $x \mapsto[x]$ is an orbital morphism from $G$ onto $G_{B}$ with recomposition $q_{[x]}=\int_{B^{-}} p_{\beta(x)} d \beta$; see Jewett $[8,13.3]$.

We next indicate how the set $\hat{G}_{B}$ of characters of $G_{B}$ can be identified with the set $\mathfrak{X}_{B}$ of $B$-characters on $G$ defined and studied by Mosak [9, §2]. Each $B$-character $\varphi$ on $G$ is constant on the $B^{-}$-orbits of $G$ and so may be regarded as a continuous function $\varphi^{B}$ on $G_{B}: \varphi^{B}([x])=\varphi(x)$ for $x \in G$. The $B$ character formula

$$
\varphi(x) \varphi(y)=\int_{B^{-}} \varphi(\beta(x) y) d \beta, \quad x, y \in G,
$$

in $[9,4.4]$ trivially implies the character formula $\varphi^{B}([x] *[y])=$ $\varphi^{B}([x]) \varphi^{B}([y])$ on the hypergroup $G_{B} \cdot \varphi^{B}$ is hermitian on $G_{B}$ because $\varphi$ is positive-definite on $G$. Thus $\varphi \mapsto \varphi^{B}$ maps $\mathfrak{X}_{B}$ into $\hat{G}_{B}$. Now consider any $\psi$ in $\hat{G}_{B}$ and define $\varphi(x)=\psi([x])$ for $x \in G$. It is easy to verify that $\varphi$ is a bounded continuous $B$-invariant function on $G$ satisfying the $B$-character formula. To conclude that $\varphi$ belongs to $\mathfrak{X}_{B}$ we need $\varphi$ to be positive-definite, which does not appear to follow directly even though $\psi$ is positive-definite on $G_{B}[8,12.3 \mathrm{~A}]$. However, a theorem of Hulanicki $[9,4.12]$ shows that $\varphi \in \mathfrak{X}_{B}$. Since $\varphi^{B}=\psi$, we conclude that $\varphi \mapsto \varphi^{B}$ maps $\mathfrak{X}_{B}$ onto $\hat{G}_{B}$. This identification is a homeomorphism since both spaces are given the topology of uniform convergence on compact sets.

Unfortunately, there exist [three-element] commutative hypergroups $K$ such that $\hat{K}$ is not a hypergroup under pointwise multiplication; see $\$ 2$. It is not even clear whether $\hat{G}_{B}$, alias $\mathfrak{X}_{B}$, must be a hypergroup. It is a hypergroup if $G$ itself is abelian, because it turns out that the notation $\hat{G}_{B}$ is unambiguous: $\left(G_{B}\right) \cong(\hat{G})_{B}$ where $\hat{G}$ denotes the character group of $G$. Incidentally, this case is of genuine interest; see $[12, \S 3]$ and $\S 6$.

Another case in which $\hat{G}_{B}$ is a hypergroup arises as follows. Let $G$ be a $Z$-group, i.e. a locally compact group such that $G / Z$ is compact where $Z$ denotes the center of $G$. Then the inner automorphism group $I(G)$ is 
compact, and $G_{I}=G_{I(G)}$ is the hypergroup of all conjugacy classes in $G$. The space $\hat{G}_{I}$ is identified with the space $\mathfrak{X}$ of normalized characters of $G$ [6]. In $\$ 5$ we will show that $\mathfrak{X}$ is a hypergroup.

2. The character hypergroup. Recall that $K$ denotes a commutative hypergroup. We say that $\hat{K}$ is a hypergroup provided it is a hypergroup with respect to pointwise multiplication. In other words, we require that the inverse Fourier-Stieltjes transform of $p_{\varphi} * p_{\psi}$ equal the pointwise product. That is,

$$
\varphi(x) \psi(x)=\int_{\hat{K}} \chi(x) d\left(p_{\varphi} * p_{\psi}\right)(\chi) \text { for } x \in K
$$

We will assume that $\hat{K}$ is a hypergroup only when necessary. Three-element hypergroups $K$ such that $\hat{K}$ is not a hypergroup are given in $[3,4.8],[8,9.1 C]$.

Dunkl, Jewett and Spector probably all knew our first lemma.

\subsection{LEMMA. $\hat{K}$ separates the points of $K$.}

Proof. Applying 12.2B of [8] with $a=0$, we see that if $\mu \in M(K)$ and $\hat{\mu}=0$, then $\mu=0$. If $x, y$ are distinct elements in $K$, then $p_{x}-p_{y} \neq 0$ and so $\left(p_{x}-p_{y}\right)^{\wedge} \neq 0$. This implies that $\psi(x) \neq \psi(y)$ for some $\psi \in \hat{K}$.

Unlike the group case, $\hat{K}$ need not span an algebra of functions on $K$. This can be seen using the hypergroup in $[8,9.3]$. Because of this, the proof of the next theorem is a little more delicate than might have been anticipated.

2.2. THEOREM. Suppose that $\hat{K}$ is a hypergroup and let $F$ be a compact subset of $K$. Then $\left.\hat{K}\right|_{F}$ spans a uniformly dense subspace of $C(F)$.

Proof. Let $S$ denote the linear span of $\left.\hat{K}\right|_{F}$ in $C(F)$. We will show that its uniform closure $S^{-}$equals $C(F)$.

Clearly $S^{-}$is a linear subspace of $C(F)$. Since $\hat{K}$ is closed under conjugation, the same is true for $S$ and for $S^{-}$. Since $\hat{K}$ separates points of $K$, $S^{-}$separates points of $F$. Since $S^{-}$also contains the constant functions, the Stone-Weierstrass theorem would apply provided $S^{-}$is closed under pointwise products. A standard approximation argument shows that it suffices to prove

$$
\varphi, \psi \in \hat{K} \text { imply }\left.\varphi \psi\right|_{F} \in S^{-} \text {. }
$$

To show this, let $\mu=p_{\varphi} * p_{\psi}$ and let $E$ be the [compact] support of $\mu$. Then

$$
\varphi(x) \psi(x)=\int_{E} \chi(x) d \mu(\chi) \text { for all } x \in K .
$$

Given $\varepsilon>0$, there is a Borel partition $\left\{E_{1}, \ldots, E_{m}\right\}$ of $E$ such that

$$
x \in F, \chi_{1}, \chi_{2} \in E_{j} \text { imply }\left|\chi_{1}(x)-\chi_{2}(x)\right|<\varepsilon .
$$

For $j=1,2, \ldots, m$, select $\psi_{j}$ in $E_{j}$. Then for $x \in F$, we have 


$$
\begin{aligned}
\left|\varphi(x) \psi(x)-\sum_{j=1}^{m} \mu\left(E_{j}\right) \psi_{j}(x)\right| & =\left|\sum_{j=1}^{m} \int_{E_{j}} \chi(x) d \mu(x)-\sum_{j=1}^{m} \int_{E_{j}} \psi_{j}(x) d \mu(\chi)\right| \\
& <\varepsilon \mu(E)=\varepsilon .
\end{aligned}
$$

Since $\left.\sum_{j=1}^{m} \mu\left(E_{j}\right) \psi_{j}\right|_{F}$ belongs to $S$ and since $\varepsilon>0$ is arbitrary, we conclude that $\left.\varphi \psi\right|_{F}$ is in $S^{-}$, i.e. (1) holds.

2.3. Corollary. Suppose that $\hat{K}$ is a hypergroup and let $H$ be a compact subhypergroup of $K$. Then every character of $H$ extends to a character of $K$.

Proof. It is obvious that restrictions of characters are characters. The theorem shows that $\left.\hat{K}\right|_{H}$ spans a uniformly dense subspace of $C(H)$, and $\hat{H}$ is an orthogonal basis for $L^{2}(H)$ by $[3,3.5]$ or 4.7 below. These two facts imply that $\left.\hat{K}\right|_{H}=\hat{H}$; compare [7, 23.20].

The following will be useful later.

\subsection{Proposition. If $\psi$ is in $\hat{K}$ and $\psi \neq 1$, then $\inf \{\operatorname{Re} \psi(z): z \in K\}<0$.}

Proof. Select $x \in K$ so that $a=\psi(x) \neq 1$ and observe that $|a|<1[8$, 6.3D]. Let $\mu_{n}$ be $p_{x} * p_{x} * \cdots * p_{x}$ ( $n$ factors). A straightforward induction argument shows that $\int_{K} \psi d \mu_{n}=a^{n}$ and, hence, $\operatorname{Re}\left(a^{n}\right)=\int_{K} \operatorname{Re} \psi(z) d \mu_{n}(z)$. If $k=\inf \{\operatorname{Re} \psi(z): z \in K\}$, the last identity implies that $k<\operatorname{Re}\left(a^{n}\right)$ for all $n$. If $|a|=1$, then $\left\{a^{n}: n=1,2, \ldots\right\}$ is a subsemigroup of the circle group and so $\operatorname{Re}\left(a^{n}\right)<0$ for some $n$. If $|a|<1$, then $\lim _{n \rightarrow \infty} \operatorname{Re}\left(a^{n}\right)=0$. In either case, $k \leqslant 0$.

It is possible that the inequality in 2.4 is always strict. However, the number " 0 " cannot be replaced by any negative number; see $[4,3.7]$.

The following corollary is well known [14, II. 2.6].

\subsection{Corollary. If $K$ is compact, then $\hat{K}$ is discrete.}

Proof. $\left\{\psi \in \hat{K}:|\psi(z)-1|<\frac{1}{2}\right.$ for all $\left.z \in K\right\}=\{1\}$.

The proof of the next proposition is similar to that of 2.4. Of course, Lemma 2.1 is also needed in the proof.

2.6. Proposition. Suppose that $\hat{K}$ is a hypergroup. If $x \in K$ and $x \neq e$, then $\inf \{\operatorname{Re} \chi(x): \chi \in \hat{K}\}<0$.

3. The center of a hypergroup. Dunkl $[3,1.6]$ defines the center $Z=Z(K)$ of a hypergroup $K$ as the set of all $x$ in $K$ such that $\operatorname{supp}\left(p_{x} * p_{y}\right)$ is a singleton for each $y \in K$. Jewett $[8,10.4]$ defines the maximum subgroup of $K$ as the set of all $x$ in $K$ such that $\operatorname{supp}\left(p_{x} * p_{\ddot{x}}\right)=\{e\}$. It is easy $[8,10.4 \mathrm{~B}]$ to show that Dunkl's center is exactly Jewett's maximum subgroup. Both terms are eminently suitably. In fact, both authors prove that $Z$ is a locally compact [abelian] group. On the other hand, if $G$ is a $Z$-group with center $Z(G)$ and if 
$x \mapsto[x]$ is the natural mapping of $G$ onto the hypergroup $G_{I}$, then $Z\left(G_{I}\right)$ is exactly the set $\{[z]: z \in Z(G)\}$. Thus $Z(K)$ for a hypergroup $K$ can be viewed as a generalized center.

For elements $x \in K$ and $z \in Z=Z(K)$, we will write $x z$ for the unique element satisfying $p_{x} * p_{z}=p_{x z}$. Likewise, for sets $A \subseteq K$ and $B \subseteq Z$ we write $A B$ in place of $A * B$.

The next result is given by Dunk1 [3, 2.2].

3.1. Proposition. Let $T_{0}$ be a closed subgroup of the circle group, and let $S=\left\{x \in K: \varphi(x) \in T_{0}\right\}$ for some $\varphi \in \hat{K}$. Then

(i) $S$ is a closed subhypergroup of $K$.

(ii) $\varphi$ is constant with value $\varphi(x) \varphi(y)$ on $\operatorname{supp}\left(p_{x} * p_{y}\right)$ for each $x, y \in S$.

(iii) If $\varphi, \psi \in \hat{K}$ and $|\varphi|=1$, then $\varphi \psi \in \hat{K}$.

3.2. Remarks. (a) If $\hat{K}$ is a hypergroup, then $Z(\hat{K})$ is, by Jewett's definition, the set $\{\varphi \in \hat{K}: \varphi \bar{\varphi}=1\}=\{\varphi \in \hat{K}:|\varphi|=1\}$. Even if $\hat{K}$ is not a hypergroup, this is a reasonable definition for $Z(\hat{K})$. Moreover, this set is also $Z(\hat{K})$ by Dunkl's definition in view of 3.1(iii).

(b) For $E \subseteq \hat{K}$, we define

$$
E^{\perp}=\{x \in K: \varphi(x)=1 \text { for all } \varphi \in E\},
$$

and for $F \subseteq K$, we define

$$
F^{\perp}=\left\{\varphi \in \hat{K}:\left.\varphi\right|_{F}=1\right\} .
$$

For $E \subseteq \hat{K}, E^{\perp}$ is a closed subhypergroup of $K$. If $\hat{K}$ is a hypergroup, then $F^{\perp}$ is a closed subhypergroup of $\hat{K}$ for $F \subseteq K$.

The next two propositions are proved by Dunkl $[3,3.12,3.13]$ for compact hypergroups. The proof of Proposition 3.3 is just like Dunkl's and we omit it, but part of our proof of Proposition 3.4 is different.

3.3. Proposition. If $F=\bigcup\left\{\operatorname{supp}\left(p_{x} * p_{\grave{x}}\right): x \in K\right\}$, then $F^{\perp}=Z(\hat{K})$.

3.4. Proposition. Suppose that $\hat{K}$ is a hypergroup and let $E=$ $\cup\left\{\operatorname{supp}\left(p_{\varphi} * p_{\bar{\varphi}}\right): \varphi \in \hat{K}\right\}$. Then

(i) $E^{\perp}=Z(K)=\{x \in K:|\varphi(x)|=1$ for all $\varphi \in \hat{K}\}$ and

(ii) $Z^{\perp \perp}=Z$.

Proof. Let $F=\{x \in K:|\varphi(x)|=1$ for all $\varphi \in \hat{K}\}$. For $x \in K$ and $\varphi \in \hat{K}$ we have

$$
|\varphi(x)|^{2}=\varphi(x) \overline{\varphi(x)}=\int_{\hat{K}} \chi(x) d\left(p_{\varphi} * p_{\bar{\varphi}}\right)(\chi) .
$$

If $x$ is in $F$ this integral is 1 and so $\chi(x)=1$ for all $\chi$ in $\operatorname{supp}\left(p_{\varphi} * p_{\bar{\phi}}\right)$. Hence $x$ is in $E^{\perp}$. On the other hand, if $x$ is in $E^{\perp}$, then the integral in (1) must equal 1 and so $|\varphi(x)|=1$, i.e. $x$ belongs to $F$. Thus we have $E^{\perp}=F$. 
For $x \in K$ and $\varphi \in \hat{K}$ we also have

$$
|\varphi(x)|^{2}=\varphi(x) \overline{\varphi(x)}=\varphi(x) \varphi(\check{x})=\int_{K} \varphi d\left(p_{x} * p_{\check{x}}\right) .
$$

If $x \in Z(K)$, then (2) shows that $|\varphi(x)|^{2}=\int_{K} \varphi d p_{e}=\varphi(e)=1$ and so $x \in F$. Thus $Z(K) \subseteq F$. If $x \in F$, then (2) shows that $\int_{K} \varphi d\left(p_{x} * p_{\dot{x}}\right)=1$ for all $\varphi \in \hat{K}$. In other words, the inverse Fourier-Stieltjes transforms of $p_{x} * p_{\dot{x}}$ and $p_{e}$ are equal on $\hat{K}$. Therefore $p_{x} * p_{\dot{x}}=p_{e}$ and so $x \in Z(K)$. Thus $F \subseteq Z(K)$ and (i) has been established.

Obviously $Z \subseteq Z^{\perp \perp}$. If $\varphi$ is in $\hat{K}$ and $x$ is in $Z$, then $|\varphi(x)|=1$ and so (1) shows that $\chi(x)=1$ for all $\chi$ in $\operatorname{supp}\left(p_{\varphi} * p_{\bar{\varphi}}\right)$. This shows that $E \subseteq Z^{\perp}$ and consequently $Z^{\perp \perp} \subseteq E^{\perp}=Z$. Thus (ii) holds.

We now define a very useful map $\lambda$; see, for example, $[3,3.15]$ and $[5,1.4]$. For $\psi \in \hat{K}$, let $\lambda(\psi)$ be the restriction of $\psi$ to $Z=Z(K)$. It is easy to verify that $\lambda$ is a continuous mapping of $\hat{K}$ into the character group $\hat{Z}$ of $Z$. Sometimes $\lambda$ maps $\hat{K}$ onto. $\hat{Z}$. This happens if $Z$ is compact by Corollary 2.3 and also if $K=G_{I}$ for a $Z$-group $G$ by a highly nontrivial theorem of Grosser and Moskowitz $[6,5.5]$, In any case, $\lambda$ has dense range in $\hat{Z}$ as we will show in Proposition 3.6. Propositions 3.5 and 3.7 hold for compact hypergroups by Dunkl [3, 3.16, 3.17].

3.5. Proposition. For $\chi \in \lambda(\hat{K}) \subseteq \hat{Z}$, let

$$
Z_{\chi}=\left\{\varphi \in \hat{K}:\left.\varphi\right|_{z}=\lambda(\varphi)=\chi\right\},
$$

and note that $Z_{1}=Z^{\perp}$. Suppose that $\hat{K}$ is a hypergroup. For $\varphi, \psi \in \hat{K}$ we have

$$
\operatorname{supp}\left(p_{\varphi} * p_{\psi}\right) \subseteq Z_{\chi} \text { where } \chi=\left.\varphi \psi\right|_{z}
$$

Proof. For $x \in Z$ we have $|\varphi(x) \psi(x)|=1$ and also

$$
\varphi(x) \psi(x)=\int_{\hat{K}} \chi(x) d\left(p_{\varphi} * p_{\psi}\right)(\chi)
$$

It follows that $\varphi(x) \psi(x)=\chi(x)$ for all $\chi$ in $\operatorname{supp}\left(p_{\varphi} * p_{\psi}\right)$.

3.6. Proposition. If $\hat{K}$ is a hypergroup, then $\lambda$ is a continuous map of $\hat{K}$ onto a dense subgroup of $\hat{Z}$.

Proof. First we show that $\lambda(\hat{K})$ is a subgroup of $\hat{Z}$. Consider two elements in $\lambda(\hat{K})$, say $\lambda(\varphi)$ and $\lambda(\psi)$ where $\varphi, \psi \in \hat{K}$. Since $\operatorname{supp}\left(p_{\varphi} * p_{\psi}\right) \neq \varnothing$, we can select some $\xi$ in $\operatorname{supp}\left(p_{\varphi} * p_{\psi}\right)$. By Proposition 3.5, we have $\left.\xi\right|_{z}=\varphi \psi \mid Z$, i.e. $\lambda(\xi)=\lambda(\varphi) \lambda(\psi)$. Thus $\lambda(\hat{K})$ is closed under products and it is easy to show that it is also closed under inversion [conjugation]. Since $\hat{K}$ separates points of $K, \lambda(\hat{K})$ separates points of $Z$. A routine argument using annihilators now shows that $\lambda(\hat{K})$ is dense in $\hat{Z}$. 
3.7. Proposition. Suppose that $\hat{K}$ is a hypergroup. For $\varphi, \psi \in \hat{K}$ we have $\lambda(\varphi)=\lambda(\psi)$ if and only if $\varphi \in \psi * Z^{\perp}$.

Proof. If $\varphi \in \psi * Z^{\perp}$, then $\varphi$ belongs to $\operatorname{supp}\left(p_{\psi} * p_{\xi}\right)$ for some $\xi \in Z^{\perp}$ and Proposition 3.5 shows that $\lambda(\varphi)=\left.\varphi\right|_{z}=\left.\psi \xi\right|_{z}=\left.\psi\right|_{z}=\lambda(\psi)$.

Now suppose that $\lambda(\varphi)=\lambda(\psi)$ and select any $\xi$ in $\operatorname{supp}\left(p_{\varphi} * p_{\bar{\psi}}\right)$. By 3.5, we have $\left.\xi\right|_{z}=\left.\varphi \bar{\psi}\right|_{z}=\lambda(\varphi) \overline{\lambda(\psi)}=1$ and so $\xi \in Z^{\perp}$. From $[8,4.1 \mathrm{~B}]$ we see that $\varphi$ is in $\operatorname{supp}\left(p_{\psi} * p_{\xi}\right)$ and so $\varphi$ belongs to $\psi * Z^{\perp}$.

3.8. Corollary. Suppose that $\hat{K}$ is a hypergroup, $\psi \in \hat{K}$ and $\chi \in \lambda(\hat{K}) \subseteq \hat{Z}$. Then $Z_{x}=\psi * Z^{\perp}$ if and only if $\lambda(\psi)=\chi$. Hence the hypercosets $\psi * Z^{\perp}$ are pairwise disjoint.

4. $Z$-hypergroups. In this section we define and study $Z$-hypergroups. An important and motivating example is the hypergroup $G_{I}$ for a $Z$-group $G$; see §5. The double-coset space $K / / H$ of a hypergroup $K$ is itself a hypergroup provided the subhypergroup $H$ is compact $[8, \S 14]$. Otherwise the situation is generally unclear, but we will show that $K / / Z$ is always a hypergroup.

4.1. THEOREM. If $H$ is a closed subgroup of $Z$, then $K / / H$ is a hypergroup.

Proof. Recall our notational convention for $x z$ where $x \in K$ and $z \in Z$ : $p_{x z}=p_{x} * p_{z}$. Since $K$ is commutative, the double-cosets in $K / / H$ all have the form $x H=\{x z: z \in H\}$. For $x H, y H$ in $K / / H, p_{x H} * p_{y H}$ is defined by the formula

$$
\int_{K / / H} f d\left(p_{x H} * p_{y H}\right)=\int_{K} f(u H) d\left(p_{x} * p_{y}\right)(u)
$$

for $f$ in $C_{0}(K / / H)$. In order to show that (1) is well defined, we first show that

$$
\int_{K} f(u H) d\left(\mu * p_{z}\right)(u)=\int_{K} f(u H) d \mu(u)
$$

for $f \in C_{0}(K / / H), \mu \in M(K)$ and $z \in H$. We define $g(u)=f(u H)$ for $u \in K$ and calculate:

$$
\begin{aligned}
\int_{K} f(u H) & d\left(\mu * p_{z}\right)(u)=\int_{K} g(u) d\left(\mu * p_{z}\right)(u) \\
= & \int_{K} \int_{K} \int_{K} g d\left(p_{x} * p_{y}\right) d \mu(x) d p_{z}(y)=\int_{K} \int_{K} g d\left(p_{x} * p_{z}\right) d \mu(x) \\
= & \int_{K} \int_{K} g d p_{x z} d \mu(x)=\int_{K} g(x z) d \mu(x) \\
= & \int_{K} f(x z H) d \mu(x)=\int_{K} f(x H) d \mu(x) .
\end{aligned}
$$

Thus (2) holds. Now suppose that $x H=x^{\prime}(H)$ and $y H=y^{\prime} H$. Then $x^{\prime}=$ 
$z_{1} x$ and $y^{\prime}=z_{2} x$ for $z_{1}, z_{2} \in H$. Applying (2) with $\mu=p_{x} * p_{y}$ and $z=z_{1} z_{2}$, we find

$$
\begin{aligned}
\int_{K} f(u H) d\left(p_{x^{\prime}} * p_{y^{\prime}}\right)(u) & =\int_{K} f(u H) d\left(p_{x} * p_{y} * p_{z_{1} z_{2}}\right)(u) \\
& =\int_{K} f(u H) d\left(p_{x} * p_{y}\right)(u) .
\end{aligned}
$$

Therefore the definition of $p_{x H} * p_{y H}$ is unambiguous and (1) is well defined.

Lengthy, but routine, arguments show that $\left(p_{x H}, p_{y H}\right) \mapsto p_{x H} * p_{y H}$ extends uniquely to a positive-continuous bilinear mapping from $M(K / / H) \times$ $M(K / / H)$ into $M(K / / H)$ and that $K / / H$ is a hypergroup. The involution in $K / / H$ is, of course, given by $(x H)^{2}=\check{x} H$.

4.2. Definition. A commutative hypergroup $K$ is called a $Z$-hypergroup provided that $K / / Z$ is a compact hypergroup.

4.3. Proposition. $A$ hypergroup $K$ is a $Z$-hypergroup if and only if $K=C Z$ for some compact subset $C$ of $K$.

Proof. The natural mapping $\pi$ of $K$ onto $K / / Z$ is open and continuous [8, 10.3B]. If $K=C Z$ and $C$ is compact, then $\pi(C)=K / / Z$ is also compact. On the other hand, if $K / / Z$ is compact, then $K / / Z=\pi(C)$ for a compact subset $C$ of $K$; this is a topological result about continuous open mappings between locally compact spaces. Hence $K=C Z$.

We next show that $(K / / Z)^{\wedge}=Z^{\perp}$.

4.4. Proposition. Let $K$ be a $Z$-hypergroup such that $\hat{K}$ is a hypergroup. For $\varphi$ in $Z^{\perp}, \varphi^{\prime}(x Z)=\varphi(x)$ defines an element of $(K / / Z)^{\wedge} . \varphi \mapsto \varphi^{\prime}$ is a one-to-one map of $Z^{\perp}$ onto $(K / / Z)^{\wedge}$ and both hypergroups are discrete.

Proof. It is easy to see that $\varphi^{\prime}$ is well defined and continuous on $K / / Z$. Moreover,

$$
\begin{aligned}
\varphi^{\prime}(x Z * y Z) & =\int_{K / / H} \varphi^{\prime}(u Z) d\left(p_{x Z} * p_{y Z}\right)(u Z)=\int_{K} \varphi(u) d\left(p_{x} * p_{y}\right)(u) \\
& =\varphi(x * y)=\varphi(x) \varphi(y)=\varphi^{\prime}(x Z) \varphi^{\prime}(y Z) .
\end{aligned}
$$

Now $\varphi^{\prime}$ is automatically hermitian since $K / / Z$ is compact $[3,3.5]$ and so $\varphi^{\prime} \in(K / / Z)^{\wedge}$. Obviously $\varphi \mapsto \varphi^{\prime}$ is one-to-one. If $\psi \in(K / / Z)^{\wedge}$, then $\varphi(x)=$ $\psi(x Z)$ defines an element in $Z^{\perp}$ such that $\varphi^{\prime}=\psi$. Thus $\varphi \mapsto \varphi^{\prime}$ maps onto $(K / / Z)^{\wedge}$.

Corollary 2.5 shows that $(K / / Z)^{\wedge}$ is discrete. To show that $Z^{\perp}$ is discrete it suffices to show that 1 is isolated in $Z^{\perp}[8,7.1 \mathrm{~B}]$. By $4.3 K=C Z$ for a compact set $C$ in $K$. Clearly

$$
W=\left\{\varphi \in Z^{\perp}:|\varphi(c)-1|<\frac{1}{2} \text { for all } c \in C\right\}
$$


is a neighborhood of 1 in $Z^{\perp}$ and

$$
W=\left\{\varphi \in Z^{\perp}:|\varphi(x)-1|<\frac{1}{2} \text { for all } x \in K\right\}
$$

since $K=C Z$. Proposition 2.4 now shows that $W=\{1\}$ and so 1 is isolated in $Z^{\perp}$.

4.5. CoRollary. If $K$ is as in 4.4, then $(K / / Z)^{\wedge}$ is a discrete hypergroup under pointwise multiplication.

We now prove some orthogonality relations similar to those in Grosser and Moskowitz [6, §2].

4.6. THEOREM. Let $K$ be a Z-hypergroup where $\hat{K}$ is a hypergroup. If $\psi, \varphi$ are in $\hat{K}$ and $\lambda(\psi)=\lambda(\varphi)$, then $\psi \bar{\varphi}$ is constant on each $x Z$ and hence induces $a$ continuous function on $K / / Z$. Moreover,

$$
\int_{K / / Z} \psi \bar{\varphi}=0 \text { if and only if } \psi \neq \varphi \text {. }
$$

Proof. If $x \in K$ and $z \in Z$, then $\psi(x z)=\psi(x) \psi(z)$ and $\varphi(x z)=$ $\varphi(x) \varphi(z)$. Also $\psi(z)=\varphi(z)$ has modulus 1 and so

$$
\psi(x z) \overline{\varphi(x z)}=\psi(x) \overline{\varphi(x)} \psi(z) \overline{\varphi(z)}=\psi(x) \overline{\varphi(x)} .
$$

Thus $\psi \bar{\varphi}$ induces a continuous function on $K / / Z$.

Now suppose that $\psi \neq \varphi$ and that $\lambda(\psi)=\lambda(\varphi)$. Then $\left.\psi \bar{\varphi}\right|_{Z}=1$ and so $\operatorname{supp}\left(p_{\psi} * p_{\bar{\varphi}}\right) \subseteq Z_{1}=Z^{\perp}$ by Proposition 3.5. By 4.4, $Z^{\perp}$ is discrete and so $\operatorname{supp}\left(p_{\psi} * p_{\bar{\psi}}\right)$ is both compact and discrete, hence finite. Since $\psi \neq \varphi, 1$ is not in $\operatorname{supp}\left(p_{\psi} * p_{\bar{\varphi}}\right)$. Thus

$$
\operatorname{supp}\left(p_{\psi} * p_{\bar{\varphi}}\right)=\left\{\varphi_{1}, \ldots, \varphi_{n}\right\} \subseteq Z^{\perp} \backslash\{1\} .
$$

It follows that $\psi \bar{\varphi}=\sum_{j=1}^{n} a_{j} \varphi_{j}$ and so

$$
\int_{K / / Z} \psi \bar{\varphi}=\sum_{j=1}^{n} a_{j} \int_{K / / Z} \varphi_{j}^{\prime}=0
$$

since each $\varphi_{j}^{\prime}$ is a character different from 1 on the compact commutative hypergroup $K / / Z[3,3.5]$.

5. $Z$-groups and $Z$-hypergroups. Let $G$ be a $Z$-group with center $Z$, and let $G_{I}$ be the hypergroup of conjugacy classes discussed at the end of $\$ 1$. Each class [ $z]$ in $G_{I}$ consists of a single point for $z \in Z$. Hence we regard $Z \subseteq G_{I}$.

5.1. Theorem. The commutative hypergroups $G_{I} / / Z$ and $(G / Z)_{I}$ are isomorphic.

Proof. For $[x Z]$ in $(G / Z)_{I}$, we define $\varphi([x Z])=[x] Z$. To see that $\varphi$ is well defined, consider $[x Z]=[y Z]$. Then $y Z=w x w^{-1} Z$ for some $w \in G$ 
and so $y=w x w^{-1} z_{0}$ for some $z_{0} \in Z$. To show $[x] Z=[y] Z$ it suffices to show $[y] Z \subseteq[x] Z$. If $z_{1} \in Z$, then $y z_{1}=w x w^{-1} z_{0} z_{1}$ and we claim

$$
p_{[y]} * p_{z_{1}}=p_{[x]} * p_{z_{0} z_{1}} \text { in } M\left(G_{I}\right) \text {. }
$$

To see this, observe that $\beta(y z)=\beta(y) z$ for any inner automorphism $\beta \in$ $I(G), y \in G$ and $z \in Z$, and therefore

$$
\begin{aligned}
p_{[y]} * p_{z_{1}} & =\int_{I(G)} p\left[\beta(y) z_{1}\right] d \beta=\int_{I(G)} p_{\left[\beta\left(y z_{1}\right)\right] d \beta} \\
& =\int_{I(G)} p\left[\beta\left(w x w^{-1} z_{z_{0} z_{1}}\right)\right] d \beta=\int_{I(G)} p\left[\beta\left(w x w^{-1}\right) z_{z_{0} z_{1}}\right] d \beta \\
& =p_{\left[w x w^{-1}\right] * p_{z_{0} z_{1}}}=p_{[x] * p_{z_{0} z_{1}}}
\end{aligned}
$$

From (1) we infer that

$$
\operatorname{supp}\left(p_{[y]} * p_{z_{1}}\right)=\operatorname{supp}\left(p_{[x]} * p_{z_{0} z_{1}}\right) \subseteq[x] Z
$$

and hence $[y] Z \subseteq[x] Z$. Similar arguments show that $\varphi$ is one-to-one and onto, and it is straightforward to check that $\varphi$ is a homeomorphism.

To show that $\varphi$ preserves convolution it suffices to show that

$$
\int_{G_{I} / / Z} f d\left(p_{[x] z} * p_{[y] z}\right)=\int_{(G / Z)_{l}} f \circ \varphi d\left(p_{[x z]} * p_{[y z]}\right)
$$

for $f$ in $C\left(G_{I} / / Z\right)$. For $\beta \in I(G)$ and $x Z \in G / Z$ we define $\beta^{\prime}(x Z)=$ $\beta(x) Z$. One verifies easily that $\beta \mapsto \beta^{\prime}$ is a well-defined continuous group homomorphism of $I(G)$ onto $I(G / Z)$. A standard argument, using this fact and the uniqueness of Haar measure on $I(G / Z)$, shows that

$$
\int_{I(G / Z)} g(\gamma) d \gamma=\int_{I(G)} g\left(\beta^{\prime}\right) d \beta
$$

for all continuous functions $g$ on $I(G / Z)$. Now consider the natural maps:

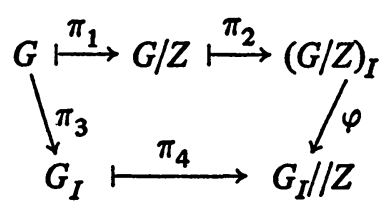

This diagram commutes: $\varphi \circ \pi_{2} \circ \pi_{1}=\pi_{4} \circ \pi_{3}$. To check (2) consider $f$ in $C\left(G_{I} / / Z\right), x, y \in G$. For $\gamma \in I(G / Z)$ we define $g(\gamma)=$ $f \circ \varphi \circ \pi_{2}(\gamma(x Z) y Z)$. Then $g$ is continuous on $I(G / Z)$ and we will apply (3) to $g$ below. Indeed, we have

$$
\begin{aligned}
& \int_{(G / Z)_{I}} f \circ \varphi d\left(p_{[x Z]} * p_{[y Z]}\right)=\int_{I(G / Z)} f \circ \varphi([\gamma(x Z) y Z]) d \gamma \\
& \quad=\int_{I(G / Z)} f \circ \varphi \circ \pi_{2}(\gamma(x Z) y Z) d \gamma=\int_{I(G)} f \circ \varphi \circ \pi_{2}\left(\beta^{\prime}(x Z) y Z\right) d \beta
\end{aligned}
$$


Now

$$
\begin{aligned}
f \circ \varphi \circ \pi_{2}\left(\beta^{\prime}(x Z) y Z\right)=f \circ \varphi \circ \pi_{2}(\beta(x) Z y Z)=f \circ \phi \circ \pi_{2}(\beta(x) y Z) \\
\quad=f \circ \varphi \circ \pi_{2} \circ \pi_{1}(\beta(x) y)=f \circ \pi_{4} \circ \pi_{3}(\beta(x) y)=f \circ \pi_{4}([\beta(x) y])
\end{aligned}
$$

and so the integrals in (4) equal

$$
\begin{aligned}
\int_{I(G)} f \circ \pi_{4}([\beta(x) y]) d \beta & =\int_{G_{I}} f \circ \pi_{4} d\left(p_{[x]} * p_{[y]}\right) \\
& =\int_{G_{I} / / Z} f d\left(p_{[x] z} * p_{[y] z}\right) .
\end{aligned}
$$

This establishes (2) as desired.

\subsection{Corollary. $G_{I}$ is a Z-hypergroup.}

As we mentioned in $\$ 1$, the set $\hat{G}_{I}$ is identified with the space $\mathfrak{X}$ of normalized characters on $G$. To be more explicit, let $G^{\wedge}$ denote the set of equivalence classes of continuous irreducible unitary representations of $G$. Each representation $\rho$ in $G^{\wedge}$ has finite dimension $d_{\rho}[6,2.1]$. If $\chi_{\rho}$ is the character of the representation $\rho$, then $\psi_{\rho}=d_{\rho}^{-1} \chi_{\rho}$ is the normalized character corresponding to $\rho$. The space $\mathfrak{X}$ of normalized characters on $G$ is exactly the space of $I(G)$-characters $[6,4.2]$ and hence is in one-to-one correspondence with $\hat{G}_{I}$. For $\rho \in G^{n}$, let $\lambda\left(\psi_{\rho}\right)=\left.\psi_{\rho}\right|_{z}$. Then $\lambda: \mathfrak{X} \mapsto \hat{Z}$ is the map used in $[6, \S 6]$ and [5] and is essentially the map we defined prior to Proposition 3.5. It will be the basic tool used to prove Theorem 5.5, which will show that $\hat{G}_{I}$ is a hypergroup.

5.3. Discussion. For $\rho, \sigma$ in $G^{\wedge}$ we have $\rho \otimes \sigma=\sum_{i=1}^{m} n_{i} \nu_{i}$ with $\nu_{i}$ in $G^{\wedge}$. Thus

$$
\chi_{\rho} \chi_{0}=\sum_{i=1}^{m} n_{i} \chi_{v_{i}}
$$

and hence

$$
\psi_{\rho} \psi_{\sigma}=\sum_{i=1}^{m} a_{i} \psi_{v_{i}} \text { where } a_{i}=n_{i} d_{\nu_{i}} d_{\rho}^{-1} d_{\sigma}^{-1} .
$$

In other words, if $\psi, \varphi$ are in $\mathfrak{X}$, then

$$
\psi \varphi=\sum_{i=1}^{m} a_{i} \psi_{i} \quad \text { where } \psi_{i} \in \mathfrak{X} \text { and } a_{i}=n_{i} d_{i} d_{\psi}^{-1} d_{\varphi}^{-1} ;
$$

here $d_{\psi}, d_{\varphi}$ and $d_{i}$ represent the dimensions of the representations corresponding to $\psi, \varphi$ and $\psi_{i}$, respectively. Note that $\sum_{i=1}^{m} a_{i}=1$. Since

$$
\lambda \dot{(\psi)} \lambda(\varphi)=\sum_{i=1}^{m} a_{i} \lambda\left(\psi_{i}\right) \text { on } Z
$$


and since characters on $Z$ are linearly independent, we see that

$$
\lambda\left(\psi_{i}\right)=\lambda(\psi) \lambda(\varphi) \text { whenever } a_{i} \neq 0 \text {. }
$$

5.4. Lemama. Consider a net $\left(\psi_{\alpha}, \varphi_{\alpha}\right)$ in $\mathfrak{X} \times \mathfrak{X}$ converging to $(\psi, \varphi)$ and expand $\psi \varphi$ as in 5.3(1). For sufficiently large $\alpha$ we have

$$
\psi_{\alpha} \varphi_{\alpha}=\sum_{i=1}^{m} a_{i} \psi_{i \alpha}
$$

where

$$
\lim _{\alpha} \psi_{i \alpha}=\psi_{i} \text { for } i=1,2, \ldots, m \text {. }
$$

Proor. Since $\rho \mapsto \psi_{\rho}$ is a homeomorphism of $G^{\wedge}$ onto $\mathfrak{X}[9,5.3]$, Theorem 1.4 of [5] and 5.5 of [6] combine to assert that $\lambda: \mathfrak{X} \mapsto \hat{Z}$ is a local homeomorphism of $\mathfrak{X}$ onto $\hat{Z}$. In view of 5.3(2), for each $i=1,2, \ldots, m$ there is a neighborhood $W_{i}$ of $\psi_{i}$ in $\mathfrak{X}$ such that $\lambda_{i}=\left.\lambda\right|_{W_{i}}$ is a homeomorphism of $W_{i}$ onto a neighborhood $U_{i}$ of $\lambda(\psi) \lambda(\varphi)$ in $\hat{Z}$. We may assume that the $W_{i}$ are pairwise disjoint and that the $U_{i}$ are all equal, to $U$ say. For large $\alpha, \lambda\left(\psi_{\alpha}\right) \lambda\left(\varphi_{\alpha}\right)$ belongs to $U$. For such $\alpha$ and each $i$, there exists $\psi_{i \alpha}$ in $W_{i}$ for which $\lambda\left(\psi_{i \alpha}\right)=$ $\lambda\left(\psi_{\alpha}\right) \lambda\left(\varphi_{\alpha}\right)$. Since the sets $W_{i}$ are disjoint, the orthogonality relations in $[6,6.1]$ show that

$$
\int_{G / Z} \psi_{i \alpha} \bar{\psi}_{j \alpha}=0 \text { for } i \neq j .
$$

From $[6,4.3]$ it follows that

$$
\int_{G / Z}\left|\psi_{i \alpha}\right|^{2}=d_{i \alpha}^{2}
$$

Likewise, we have

$$
\int_{G / Z} \psi_{i} \bar{\psi}_{j}=0 \text { for } i \neq j
$$

and

$$
\int_{G / Z}\left|\psi_{i}\right|^{2}=d_{i}^{2}
$$

Since each $\lambda_{i}$ is a homeomorphism, (2) holds. Since dimension is continuous [5, p. 67], we have $\lim _{\alpha} d_{i \alpha}=d_{i}, \lim _{\alpha} d_{\psi_{\alpha}}=d_{\psi}$ and $\lim _{\alpha} d_{\varphi_{\alpha}}=d_{\varphi}$. Since dimension is integer-valued, we have

$$
d_{i \alpha}=d_{i}, d_{\psi_{\alpha}}=d_{\psi} \text { and } d_{\varphi_{\alpha}}=d_{\varphi}
$$

for large $\alpha$. For each $\alpha$ we can write

$$
\psi_{\alpha} \varphi_{\alpha}=\sum_{i=1}^{m} a_{i \alpha} \psi_{i \alpha}+\sum_{j=1}^{k_{\alpha}} b_{j \alpha} \psi_{j \alpha}^{\prime}
$$


with all $\psi_{i \alpha}$ and $\psi_{j \alpha}^{\prime}$ distinct. Again the orthogonality relations hold $[6,6.1]$ and so

$$
a_{i \alpha} d_{i \alpha}^{2}=\int_{G / Z} \psi_{\alpha} \varphi_{\alpha} \bar{\psi}_{i} \rightarrow \int_{G / Z} \psi \varphi \overline{\psi_{i}}=a_{i} d_{i}^{2}
$$

here we used (3)-(6). Now from (7) we infer that $\lim _{\alpha} a_{i \alpha}=a_{i}$ for each $i$. For large $\alpha, a_{i \alpha}$ must have the form $k / d_{\psi} d_{\varphi}$ for some integer $k$ [see 5.3(1)] and so each $a_{i \alpha}$ equals $a_{i}$ for large $\alpha$. Since $\sum_{i=1}^{m} a_{i}=1$, we conclude that the $b_{j \alpha}$ 's in (8) do not appear for large $\alpha$ and so (8) is equivalent to (1).

5.5. THEOREM. Let $G$ be a Z-group. The space $\mathfrak{X}$ of normalized characters on $G$ has a hypergroup structure compatible with pointwise multiplication:

$$
\text { if } \psi \varphi=\sum_{i=1}^{m} a_{i} \psi_{i} \text {, then } p_{\psi} * p_{\varphi}=\sum_{i=1}^{m} a_{i} p_{\psi_{i}}
$$

Proof. It is easy to check that the convolution defined in (1) extends to an associative convolution on $M(\mathfrak{X})$. According to [8, 2.4B], convolution will be positive-continuous provided $(\psi, \varphi) \mapsto p_{\psi} * p_{\varphi}$ is continuous from $\mathfrak{X} \times \mathfrak{X}$ into $\mathrm{M}^{+}(\mathfrak{X})$. This is easily shown using Lemma 5.4. Lemma 5.4 can also be used to show that the map $(\psi, \varphi) \mapsto \operatorname{supp}\left(p_{\psi} * p_{\varphi}\right)$ is continuous from $\mathfrak{X} \times \mathfrak{X}$ into the space of compact subsets of $\mathfrak{X}$.

The identity in $\mathfrak{X}$ is the function 1 and the involution is given by conjugation. Let $\psi, \varphi$ be in $\mathfrak{X}$; we check that $1 \in \operatorname{supp}\left(p_{\psi} * p_{\bar{\varphi}}\right)$ if and only if $\psi=\varphi$. In any case we can write

$$
\psi \bar{\varphi}=c \cdot 1+\sum_{i=1}^{m} c_{i} \psi_{i} \quad \text { with all } \psi_{i} \neq 1
$$

If 1 is in $\operatorname{supp}\left(p_{\psi} * p_{\bar{\varphi}}\right)$, then $c \neq 0$ and so $\lambda\left(\psi_{i}\right)=\lambda(\psi) \overline{\lambda(\varphi)}=1$ for all $i$ by 5.3(2). Then $\int_{G / Z} \psi \bar{\varphi}=c \neq 0$ by the orthogonality relations $[6,6.1]$ and the same result shows that $\psi=\varphi$. On the other hand, if $\psi=\varphi$ then $\lambda(\psi)=\lambda(\varphi)$ and so $\lambda(\psi) \overline{\lambda(\varphi)}=1$. The orthogonality relations hold again and we find

$$
c=\int_{G / Z} \psi \bar{\varphi}=\int_{G / Z}|\psi|^{2}>0,
$$

i.e. $1 \in \operatorname{supp}\left(p_{\psi} * \mathrm{p}_{\bar{\varphi}}\right)$.

6. $M(K)$ for special $K$. For a nondiscrete locally compact abelian group $G$ the measure algebra $M(G)$ is always a very complicated object compared with $L^{1}(G)$. In contrast, for some nondiscrete hypergroups $K, M(K)$ is a nice well-behaved object. This fact has been observed for the algebra of radial measures on $\mathrm{R}^{n}[13, \S 4]$, [11] and for the center of $M(G)$ where $G$ is a compact simple Lie group [10]. Dunkl $[2, \S 3]$ studies well-behaved $M(K)$ for certain compact hypergroups $K$. In this section, we generalize Dunkl's results so that they apply, for example, to the algebra of radial measures on $\mathbf{R}^{n}$. 
Proofs will be sketchy or omitted. Several examples are discussed.

The proof of the first proposition mimics the proof for the group case [7, 20.17]. Note that every commutative hypergroup $K$ has a Haar measure $m$; this nontrivial fact was proved by Spector [15].

6.1. Proposition. If $A$ is an m-measurable subset of $K$ such that $0<m(A)$ $<\infty$, then $e$ is in the interior of $A * \check{A}$.

6.2. Corollary. If $H$ is a subhypergroup of $K$ containing a subset $A$ such that $0<m(A)<\infty$, then $H$ is open.

Proof. Use 6.1 and [8, 10.2A].

We next determine when the center $Z$ of $K$ has positive measure; cf. [2, 3.4].

6.3. Proposition. Suppose that $\hat{K}$ is a hypergroup. The following are equivalent:

(i) Haar measure $m_{Z}$ on $Z$ is a nonzero multiple of $\left.m\right|_{Z}$,

(ii) $0<m(A)<\infty$ for some subset $A$ of $Z$,

(iii) $Z$ is open in $K$,

(iv) each hypercoset $\psi * Z^{\perp}$ is compact in $\hat{K}$,

(v) $Z^{\perp}$ is compact in $\hat{K}$,

(vi) some hypercoset $\psi * Z^{\perp}$ is compact in $\hat{K}$.

Proof. The equivalence of (i)-(iii) is straightforward; see 6.2. The equivalence of (iv)-(vi) is also straightforward; see [8, 3.2B].

To see that (ii) implies (v), note that $\mu=\left.m\right|_{A}$ is absolutely continuous with respect to $m$ and so $\hat{\mu} \in C_{0}(\hat{K})$ by $[8,7.3 F]$. Hence $\{\psi \in \hat{K}: \hat{\mu}(\psi)=m(A)\}$ is compact in $\hat{K}$ and it suffices to observe that $Z^{\perp} \subseteq\{\psi \in \hat{K}: \hat{\mu}(\psi)=m(A)\}$.

Finally we show that (v) implies (iii). Let $a$ be the Haar measure on the compact hypergroup $Z^{\perp}$. Then $a \in M^{+}(\hat{K})$ and the inverse Fourier-Stieltjes transform $\check{a}$ is continuous on $K$. Thus $\{x \in K: \check{a}(x) \neq 0\}$ is open in $K$ and it suffices to prove

$$
Z=\{x \in K: \check{a}(x) \neq 0\} .
$$

Consider $x$ in $K$ and define $x^{\sharp}(\psi)=\psi(x)$ for $\psi \in Z^{\perp}$. Then $x^{\sharp}$ is a character on the hypergroup $Z^{\perp}$ and

$$
\check{a}(x)=\int_{Z^{\perp}} x^{\sharp}(\psi) d a(\psi) .
$$

The characters on $Z^{\perp}$ form an orthogonal basis for $L^{2}\left(Z^{\perp}\right)$ and so $\check{a}(x) \neq 0$ if and only if $x^{\#}=1$, i.e. if and only if $x$ is in $Z^{\perp \perp}$. So (1) holds by (3.4(ii)).

For $\mu \in M(K)$, let $\pi \mu=\left.\mu\right|_{z}$. Let $M_{h}(K)=\{\mu \in M(K):|\mu|(Z)=0\}$. 
6.4. LEMMA. $M(K)=M(Z) \oplus M_{h}(K)$ and the projection $\pi$ of $M(K)$ onto $M(Z)$ is bounded with norm 1 .

Sometimes $\pi$ is an algebra homomorphism, as we will show in Proposition 6.7. $M_{a}(K)$ denotes the set of measures in $M(K)$ that are absolutely continuous with respect to $m$. If $x \in K$ and $z \in Z$ then $p_{x} * p_{z}=p_{x z}$. But in some hypergroups it turns out that $p_{x} * p_{y} \in M_{a}(K)$ for $x, y \in K \backslash Z$. With this in mind, we will say that $K$ is $n$-fold absolutely continuous if $x_{1}, \ldots, x_{n}$ $\in K \backslash Z$ implies $p_{x_{1}}{ }^{*} \cdots * p_{x_{n}} \in M_{a}(K)$. For examples of this phenomenon, see 6.12-6.14 and 6.17.

\subsection{LEMMA. If $K$ is $n$-fold absolutely continuous, then}

$$
\mu_{1}, \ldots, \mu_{n} \in M_{h}(K) \text { implies } \mu_{1} * \ldots * \mu_{n} \in M_{a}(K) \text {. }
$$

Proof. Consider $\mu_{1}, \ldots, \mu_{n} \in M_{h}(K)$ and $\mu=\mu_{1} * \cdots * \mu_{n}$. For $k=1$, $2, \ldots, n$ let $A_{k}$ be the $n$-fold Cartesian product whose $k$ th factor is $Z$ and whose other factors are $K$. Let $A_{0}$ be the $n$-fold Cartesian power of $K \backslash Z$. Let $B$ be a Borel set in $K$ with $m(B)=0$. Then

$$
|\mu|(B)<\sum_{k=0}^{n} \iint \cdots \int_{A_{k}} p_{x_{1}} * \cdots * p_{x_{n}}(B) d\left|\mu_{1}\right|\left(x_{1}\right) \cdots d\left|\mu_{n}\right|\left(x_{n}\right) .
$$

The integral over $A_{0}$ is 0 because $K$ is $n$-fold absolutely continuous. The remaining sum is bounded by $\sum_{k=1}^{n}\left(\left|\mu_{1}\right| \times \cdots \times\left|\mu_{n}\right|\right)\left(A_{k}\right)$ and this is 0 since each $\mu_{k}$ is in $M_{h}(K)$. Thus $m(B)=0$ implies $|\mu(B)|=0$ and so $\mu \in M_{a}(K)$.

6.6. Discussion AND NOTATION. Recall from $[8,6.3]$ that $\mathfrak{X}_{b}(K)$ consists of all multiplicative functions on $K$. Thus $K^{\wedge} \subseteq \mathfrak{X}_{b}(K)$ and the inclusion can be strict $[8,9.5]$. However, we have $K^{n}=\mathfrak{X}_{b}(K)$ for a large class of hypergroups; see $[17, \S 1]$.

For $\psi \in \mathfrak{X}_{b}(K)$ and $\mu \in M(K)$, define $\tau_{\psi}(\mu)=\int_{K} \bar{\psi} d \mu$. Each $\tau_{\psi}$ belongs to the carrier space $\Delta$ of $M(K)[8,6.3 \mathrm{~A}]$ and $\psi \rightarrow \tau_{\psi}$ is a homeomorphism of $\mathfrak{X}_{b}(K)$ into $\Delta$. Hence we regard $\mathfrak{X}_{b}(K) \subseteq \Delta$. For $\mu \in M(K)$ we write $\tilde{\mu}$ for its Gelfand transform: $\tilde{\mu}(\tau)=\tau(\mu)$ for $\tau \in \Delta$. Since $\hat{K} \subseteq \mathfrak{X}_{b}(K)$, we have $\hat{K} \subseteq \Delta$ and $\left.\tilde{\mu}\right|_{\hat{K}}=\hat{\mu}$.

6.7. Proposition. Suppose that $K$ is $n$-fold absolutely continuous, that $\hat{K}$ is a hypergroup and that $m(Z)=0$. Then the projection $\pi: M(K) \mapsto M(Z)$ is an algebra homomorphism and is bounded in the $\hat{K}$-sup-norm:

$$
\left\|(\pi \mu)^{\wedge}\right\|_{\infty} \leqslant\|\hat{\mu}\|_{\infty} \text { for } \mu \in M(K) .
$$

If $\mu \in M(K)$, then

$$
\mu \in M_{h}(K) \text { if and only if } \hat{\mu} \in C_{0}(\hat{K}) \text {. }
$$

Proof. We first prove (2). If $\mu \in M_{h}(K)$, then by 6.5 its $n$-fold convolution 
$\mu^{n}$ belongs to $M_{a}(K)$. Hence $\hat{\mu}^{n}$ is in $C_{0}(\hat{K})$ and so $\hat{\mu} \in C_{0}(\hat{K})$.

Now suppose that $\hat{\mu} \in C_{0}(\hat{K})$. Since $\mu=\pi \mu+\mu_{h}$ where $\mu_{h} \in M_{h}(K)$ it suffices to show that $\pi \mu=0$. Since $(\pi \mu)^{\wedge}=\hat{\mu}-\hat{\mu}_{h}$ is in $C_{0}(\hat{K})$, it suffices to prove

$$
\nu \in M(Z) \text { and } \hat{\nu} \in C_{0}(\hat{K}) \text { imply } \nu=0 .
$$

Consider any $\chi \in \lambda(\hat{K}) \subseteq \hat{Z}$. By 3.8, $Z_{\chi}=\{\psi \in \hat{K}: \lambda(\psi)=\chi\}$ is a hypercoset of $Z^{\perp}$ and so by $6.3, Z_{\chi}$ is noncompact in $\hat{K}$. $\hat{\nu}$ is constant on $Z_{\chi}$, in fact, $\hat{\nu}(\psi)=\hat{\nu}_{Z}(\chi)$ for all $\psi \in Z_{X}$ where $\hat{\nu}_{Z}$ denotes the Fourier-Stieltjes transform of $\nu$ on $Z$. Since $Z_{x}$ is closed and noncompact, $\hat{\nu}$ must vanish on $Z_{x}$. This shows that $\hat{\nu}_{Z}=0$ on $\lambda(\hat{K})$. Since $\lambda(\hat{K})$ is dense in $\hat{Z}$ by 3.6 , we conclude that $\hat{\nu}_{Z}=0$. Thus $\nu=0$ and (3) is valid.

To prove (1) we again consider $\chi \in \lambda(\hat{K})$. Since $Z_{\chi}$ is noncompact in $\hat{K}$, it is noncompact in the compact carrier space $\Delta$ of $M(K)$. Hence the closure $Z_{\chi}^{-}$of $Z_{\chi}$ in $\Delta$ is bigger than $Z_{\chi}$. Select $\tau$ in $Z_{x}^{-} \backslash Z_{\chi}$; then $\tau$ is a limit of a net $\left\{\psi_{\alpha}\right\}$ in $Z_{x}$. Thus

$$
\tilde{\mu}(\tau)=\lim _{\alpha} \hat{\mu}\left(\psi_{\alpha}\right)=\lim _{\alpha}\left[(\pi \mu)^{\wedge}\left(\psi_{\alpha}\right)+\left(\mu_{h}\right)^{\wedge}\left(\psi_{\alpha}\right)\right] .
$$

The first equality in (4) shows that

$$
|\tilde{\mu}(\tau)| \leqslant\|\hat{\mu}\|_{\infty} .
$$

We also have $(\pi \mu)^{\wedge}\left(\psi_{\alpha}\right)=(\pi \mu)^{\wedge}(\chi)$ for each $\alpha$ and $\lim _{\alpha}\left(\mu_{h}\right)^{\wedge}\left(\psi_{\alpha}\right)=0$ since $\hat{\mu}_{h} \in C_{0}(\hat{K})$. Therefore

$$
\tilde{\mu}(\tau)=(\pi \mu)^{\wedge}(\chi)
$$

and so (5) shows that

$$
\left|(\pi \mu)^{\wedge}(\chi)\right|<\|\hat{\mu}\|_{\infty} .
$$

Since (6) holds for all $\mu \in M(K)$, one readily verifies that

$$
(\pi(\mu * \nu))^{\wedge}(\chi)=(\pi \mu)^{\wedge}(\chi)(\pi \nu)^{\wedge}(\chi) .
$$

Since (7) and (8) hold for all $\chi$ in the dense subgroup $\lambda(\hat{K})$ of $\hat{Z}$, we conclude that (1) holds and that $\pi(\mu * \nu)^{\wedge}=(\pi \mu)^{\wedge}(\pi \nu)^{\wedge}$ on $\hat{Z}$. Since Fourier-Stieltjes transforms are unique, we obtain $\pi(\mu * \nu)=(\pi \mu) *(\pi \nu)$ so that $\pi$ is an algebra homomorphism.

6.8. Corollary. With the hypotheses in $6.7, M_{h}(K)$ is a closed L-ideal in $M(K)$.

The next theorem tells us that for certain hypergroups $K$ the carrier space $\Delta_{M(K)}$ of $M(K)$ is only slightly more complicated than the carrier space $\Delta_{M(Z)}$ of $M(Z)$. In particular, if $Z$ is finite, $\Delta_{M(K)}$ is very well behaved.

6.9. TheOREM. Suppose that $K$ is $n$-fold absolutely continuous, that $\hat{K}=$ $\mathfrak{X}_{b}(K)$, that $\hat{K}$ is a hypergroup and that $m(Z)=0$. As usual, we regard 
$\hat{K} \subseteq \Delta_{M(K)}$. The algebra homomorphism $\pi: M(K) \mapsto M(Z)$ induces an embedding of $\Delta_{M(Z)}$ in $\Delta_{M(K)}$. Then

$$
\hat{K} \cap \Delta_{M(Z)}=\varnothing, \quad \hat{K} \cup \Delta_{M(Z)}=\Delta_{M(K)} .
$$

The closure of $\hat{K}$ in $\Delta_{M(K)}$ equals $\hat{K} \cup \hat{Z}^{-}$where $\hat{Z}^{-}$is the closure of $\hat{Z}$ in $\Delta_{M(Z)} . A$ net $\left\{\psi_{\alpha}\right\}$ in $\hat{K}$ converges to $\tau \in \hat{Z}^{-}$if and only if it is eventually outside of each compact subset of $\hat{K}$ and $\lambda\left(\psi_{\alpha}\right) \rightarrow \tau$ in $\Delta_{M(Z)}$.

Proof. We leave the technical, but routine, details to the reader. Here is why $\hat{K}$ and $\Delta_{M(z)}$ are disjoint. If $\psi$ were in $\hat{K}$ and the corresponding functional $\tau_{\psi}$ were in $\Delta_{M(Z)}$, then $\tau_{\psi}$ would vanish on $M_{h}(K)$ and so $\psi$ would vanish on the dense set $K \backslash Z$.

To see that $\Delta_{M(K)}=\hat{K} \cup \Delta_{M(Z)}$, consider $\tau$ in $\Delta_{M(K)} \backslash \hat{K}$. Then $\tau$ vanishes on $M_{a}(K)$ by $[8,6.3 F]$ since $\hat{K}=\mathfrak{X}_{b}(K)$. Because of $6.5, \tau$ must vanish on $M_{h}(K)$. Hence $\tau$ is induced by an element of $\Delta_{M(z)}$.

6.10. COROLLARY. Under the hypotheses of 6.9, the following are equivalent:

(i) $Z$ is discrete,

(ii) $\hat{K}$ is dense in $\Delta_{M(K)}$,

(iii) $M(K)$ is a symmetric Banach algebra.

The corresponding result for locally compact abelian groups can be found in $[16,1.3 .3]$.

6.11. EXAMPLE. Let $G$ be a locally compact abelian group and let $B$ be a compact group of automorphisms of $G$ so that $G$ is an $[F I A]_{B}^{-}$-group; see $\$ 1$. The space $G_{B}$ of $B$-orbits is a commutative hypergroup. Also $\hat{G}_{B}$ is a hypergroup and $\hat{G}_{B}=\mathfrak{X}_{b}\left(G_{B}\right)$. The center of $G_{B}$ consists exactly of the one-element orbits. Thus the center of $G_{B}$ is the image of $Z_{B}=\{x \in G$ : $\beta(x)=x$ for all $\beta \in B\}$ under the natural map $\pi: G \mapsto G_{B}$. If $Z_{B}$ has Haar measure 0 in $G$, then $\pi\left(Z_{B}\right)$ has $m$-measure 0 in $G_{B}$.

For $x \in G$, let $\mu_{x}$ be the measure in $M(G)$ defined by $\int_{G} f d \mu_{x}=$ $\int_{B} f(\beta(x)) d \beta$. If $\mu_{x} * \mu_{y}$ belongs to $M_{a}(G)$ for all $x, y \in G \backslash Z_{B}$, then the hypergroup $G_{B}$ is 2 -fold absolutely continuous. It follows that $G_{B}$ satisfies all the hypotheses of Theorem 6.9 [with $n=2$ ] provided that

(i) $Z_{B}$ has Haar measure 0 in $G$,

(ii) $\mu_{x} * \mu_{y} \in M_{a}(G)$ for $x, y \in G \backslash Z_{B}$.

6.12. ExAMPLE. Let $G=\mathbf{R}^{n}$ and let $B$ be the group $O(n)$ of rotations in $\mathbf{R}^{n}$. Then $Z_{B}=\{0\}$. Ragozin [11, 2.6] shows that $\mu_{x} * \mu_{y} \in M_{a}\left(\mathbf{R}^{n}\right)$ for nonzero $x, y$ in $\mathbf{R}^{n}$. Thus (i) and (ii) in 6.11 hold. $G_{B}$ is homeomorphic with $[0, \infty), \hat{G}_{B}$ is also homeomorphic with $[0, \infty)$ and the carrier space of $M\left(G_{B}\right)$ is homeomorphic with $[0, \infty]$. Of course, these facts can be established directly $[13,4.4],[11,3.3]$.

6.13. EXAMPLE. Let $G$ be the compact group $\Delta_{p}$ of $p$-adic integers and let $B$ 
be the group of units in $\Delta_{p}$ acting multiplicatively on $\Delta_{p}$. The hypergroup $G_{B}$ is studied by Dunkl and Ramirez [4]. $G_{B}$ is identified with the set $\{0,1,2, \ldots, \infty\}$. Its center $\{\infty\}$ has $m$-measure 0 . For finite $m, n$ in $G_{B}$, $p_{m} * p_{n}(\infty)=0$ and so $p_{m} * p_{n} \in M_{a}\left(G_{B}\right)$. So Theorem 6.9 and its corollary apply to $G_{B} . \hat{G}_{B}$ can be identified with $\{0,1,2, \ldots\}$ in which case the carrier space of $M\left(G_{B}\right)$ is $\{0,1,2, \ldots, \infty\}$.

6.14. EXAMPLE. Let $G$ be the group $\Omega_{p}$ of $p$-adic numbers and again let $B$ be the group of units in $\Delta_{p} \subseteq \Omega_{p}$. Then $G_{B}=\{\ldots,-2,-1,0,1,2, \ldots, \infty\}$ and its center is $\{\infty\} . \hat{G}_{B}$ can be identified with $\{-\infty, \ldots,-2,-1,0,1$, $2, \ldots\}$; here $-\infty$ corresponds to the character identically equal to 1 . The carrier space of $M\left(G_{B}\right)$ is the one-point compactification of $G_{B}^{\wedge}$, i.e. the two-point compactification of the integers.

6.15. REMARK. The facts that $n$ is an integer in Example 6.12 and that $p$ is an integer in Example 6.13 are too restrictive. Extensions of these examples are studied by Alan Schwartz [13] and Dunkl and Ramirez [4], respectively.

6.16. EXAMPLE. Dunkl [1] begins with the $k$-dimensional sphere $S^{k}, k>2$, which has a unique rotation-invariant measure. Let $p$ be a fixed "north pole" in $S^{k}$ and let $G_{p}=\{\alpha \in S O(k+1): \alpha(p)=p\}$. The $G_{p}$-invariant functions and measures on $S^{k}$ are called zonal functions and zonal measures. Since they are determined by their values on $G_{p}$-orbits, one may transfer them to the space $K=S^{k} / G_{p}$ of $G_{p}$-orbits, which is homeomorphic with [-1,1]. The center of $K$ corresponds to the trivial orbits [the north and south pole], i.e. to $\{-1,1\}$. It turns out that $K$ is a hypergroup to which 6.9 and 6.10 apply. $\hat{K}$ can be identified with $\{0,1,2,3, \ldots\}$. The carrier space of $M(K)$ has two more elements, $\tau_{E}$ and $\tau_{0} ; \tau_{E}=\lim _{n \rightarrow \infty} 2 n$ and $\tau_{0}=\lim _{n \rightarrow \infty}(2 n+1)$.

6.17. EXAMPLE. Let $G_{I}$ be the space of conjugacy classes of a compact connected simple Lie group $G$. Ragozin [10] observes that the center of $G$ is finite and so the center of $G_{I}$ is also finite. From $[10,2.2]$ it follows that $G_{I}$ is $n$-fold absolutely continuous where $n$ is the dimension of $G$. So again 6.9 and 6.10 apply. If $G=S U(2)$, then $G_{I}=[0,2 \pi]$ and its center is $\{0,2 \pi\}$. If $G=S O(3)$, then $G_{I}=[0, \pi]$ and its center is $\{0\}$.

\section{REFERENCES}

1. C. F. Dunkl, Operators and harmonic analysis on the sphere, Trans. Amer. Math. Soc. 125 (1966), 250-263.

2. ___ Structure hypergroups for measure algebras, Pacific J. Math. 47 (1973), 413-425.

3. The measure algebra of a locally compact hypergroup, Trans. Amer. Math. Soc. 179 (1973), 331-348.

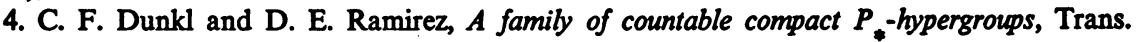
Amer. Math. Soc. 202 (1975), 339-356.

5. S. Grosser, R. Mosak and M. Moskowitz, Duality and harmonic analysis on central topological groups. I, II, Indag. Math. 35 (1973), 65-91.

6. S. Grosser and M. Moskowitz, Representation theory of central topological groups, Trans. Amer. Math. Soc. 129 (1967), 361-390. 
7. E. Hewitt and K. A. Ross, Abstract harmonic analysis. I, II, Springer-Verlag, New York, $1963,1970$.

8. R. I. Jewett, Spaces with an abstract convolution of measures, Advances in Math. 18 (1975), $1-101$.

9. R. D. Mosak, The $L^{1}$ - and $C^{*}$-algebras of $[F I A]_{B}^{-}$groups, and their representations, Trans. Amer. Math. Soc. 163 (1972), 277-310.

10. D. L. Ragozin, Central measures on compact simple Lie groups, J. Functional Analysis 10 (1972), 212-229.

11. Rotation invariant measure algebras on Euclidean space, Indiana Univ. Math. J. 23 (1974), 1139-1154.

12. K. A. Ross, Hypergroups and centers of measure algebras, Ist. Naz. Alta Mat. (Symposia Math.) 22 (1977), 189-203.

13. A. Schwartz, The structure of the algebra of Hankel transforms and the algebra of Hankel-Stieltjes transforms, Canad. J. Math. 23 (1971), 236-246.

14. R. Spector, Apercu de la theorie des hypergroupes, Lecture Notes in Math., vol. 497, Springer-Verlag, Berlin. (Analyse Harmonique sur les Groupes de Lie, Sem. Nancy-Strasbourg 1973-1975).

15. Mesures invariantes sur les hypergroupes (to appear).

16. J. L. Taylor, Measure algebras, CBMS Regional Conf. Ser. in Math., no. 16, Amer. Math. Soc. Providence, R.I., 1973.

17. A. K. Chilana and K. A. Ross, Spectral synthesis in hypergroups, Pacific J. Math. 74 (1978).

Department of Mathematics, University of Oregon, Eugene, Oregon 97403 